\title{
Research on Translation Skills of English Literature Works from the Perspective the Culturology
}

\author{
Wu Chen \\ School of Foreign Languages, Xi'an Shiyou University, Xi'an, Shanxi. 710065
}

Keywords: the perspective of culturology, English literature works, translation skills

\begin{abstract}
With the increasingly frequent international exchanges, the importance of translation skills is rising. The literature works have no nationality, but the language has the national boundaries. Seen from the overall tendency of the literature development, translation has gradually formed an independent discipline. Based on the perspective of culturology, the translation of English literature works must be completed according to the theories and principles, so as to ensure the translation results to retain the literature contents and improve the translation effects. This paper makes a detailed analysis of translation skills of the English literature works from the perspective of culturology, hoping to provide a theoretical reference for the related workers.
\end{abstract}

\section{Introduction}

With the internationalized literature development, the world literature shows a significant development tendency; the cultural exchanges also become increasingly frequent. The literature works of the Chinese language, with the biggest using population and the mostly widely applied English language are also frequently translated, which also indirectly improves the importance of the translation skills of Chinese and English literature works. However, out of the cultural differences, it is difficult to realize the unity of "spirit and form". Therefore, it is of the significant realistic meaning to discuss the translation skills of English literature works from the perspective of culturology.

\section{Basic Ideas of Culturology and the Translation of Literature Works}

Seen from the basic concepts of literature, culturology mainly refers to a discipline that studies the cultural characteristics of the disciplines. There is the close and inseparable correlation between culturology and the language development [1]. The expression of language and speech will be obviously influenced by the cultural developments in different periods and regions. Therefore, all the cultural and artistic activities of culturology are the basic contents, and meanwhile characterized by the regional, diversified, traditional, epochal and practical features [2].

Translation is a discipline retained by the language translation and art. The translation requirements of the literature works should have certain roles, translation nature, standards and principles. Meanwhile, in the process of actual translation, explore the humanity and art characteristics of the literature works under the different language backgrounds, so as to guarantee the final translation quality [3].

\section{Basic Requirements for the Literature Works Translation from the Perspective of Culturology}

From the perspective of culturology, the translation of literature works must be based on the mutual conversion between English and Chinese. Meanwhile, the English literature works must be established on the trend basis of culture [4]. In order to finish the translation work of the English literature works on the basis of expression culturology, we have to firstly analyze the cultural differences, convert our thoughts of the cultures in our country and other English-speaking countries from the perspective of culturology and then conduct cultural discussion and integration. 
Therefore, in the translation process, we must pay attention whether we can describe and express the verb-centered sentences from the perspective of the thoughts of Chinese users; the sentences are simple, which can be realized through a certain mode while expressing the emotions [5]. Meanwhile, most of the sentences in the English system are mainly the subject plus predicate in terms of narration, mixed with some phrases and clauses. Therefore, in terms of sentence type, the English system is accustomed to using the long sentence. The long sentence is complicated and its narration ability is similar to the characteristics of "the scattered form and the lingering spirits". Second, accurately master the differences of cultural accumulations in our country and the English-speaking countries. For example, from the perspective of the number, our country regards 6 and 8 as the lucky numbers. However, in the English cultural system, the number 8 is seldom used; meanwhile, influenced by the Bible, more people like to use "7" and meanwhile contradict against 13 and Friday. Therefore, in the translation process of the English literawture works, we need to pay attention to the tradition of cultures and promote the translation result to maintain the according emotions. Through the analysis of the works, it is found that "Naplcs" also indicates the south port city in Italy, with the beautiful scenery. Therefore, the cultural background of English works needs to be mastered in the translation process, so as to translate it. Finally, we also need to emphasize the habits of language expression. Take the time as example. In the word system in our country, we mainly express time through YY MM DD. Therefore, from the perspective of culturology, the translation characteristics and skills of English literature works must be done from the cultural perspective.

\section{Translation Principles and Skills of English Literature Works from the Perspective of Culturology}

The translation of English literature works from the perspective of culturology must start from the literary value and social value of the English works itself. Therefore, the skill characteristics of translation must be fully shown in the translation process and the cultural contents in the original text must be retained to the fullest, so as to realize the translation effect of "faithfulness, elegance and expressiveness".

\subsection{Translation Principle}

The Chinese language and the English languages are the two most important language forms in the international society, with wide users and application scope. However, influenced by multiple factors like the living habits, natural environment, social history, the two languages vary a lot in terms of the language form and the cultural form. Meanwhile, they also have obvious differences in terms of the language use background. Therefore, in the translation process, several basic principles must be followed. First of all, translate according to the basic premise of the translation of literature works, respect the cultural background differences and the language development differences of both sides and guarantee that the translators can accurately and comprehensively master the cultural contents that the English literature works want to express. Second, follow the entirety and unity of the English literature works in terms of cultural style. For example, in the $18^{\text {th }}$ century, romanticism prevailed, with a large number of English literature works; the classical literature was popular in the English creation process. Therefore, rhyme and thirteen-line poems appeared a lot in the works. Therefore, in the translation process, try to adhere to the basic style of romanticism and retain the representation mode of rhyme.

\subsection{Translation Skills}

The translation of English literature works from the perspective of culturology is bound to suffer from many obstacles. Therefore, in the translation process, we must fully master the development conditions of culturology and the basic ideas of literary translation. And adopt the special translation styles according to the characteristics of the literary works [6]. For example, in the translation version of Norwegian Wood of Lin Shaohua, Haruki Murakami thought highly of him and held that the translated version was more excellent than the original version. Therefore, the 
translation is not the imitation process, but the creation and optimization process.

\subsubsection{Mastery of Relationship}

In the translation process, we firstly have to master the diversified relationship and guarantee the accuracy of the translation results. From the perspective of culturology, we need to make clear the following three relationships. 1 . The relationship between works and translators. The translators belong to the independent subjects and have to own certain personal likeness and literary qualities. Therefore, in the translation process of English literature works, they tend to choose the sentence forms and phrases they like for expression. Meanwhile, the translators, influenced by ideology, may neglect that the English literature works belong to the derivative literary creation under the capitalism social form. So they may go against the translation version. Therefore, the translators must translate works after properly forgetting and even getting rid of the subjective ideas. 2 . The relationship between translators and the translated works. Translation is the regeneration process of literature works, so translators must fully consider about their works' conditions in terms of the consumption ideas and readers' expectations. They must fully master the active factors, improve the use ability of both Chinese and English, so as to fully satisfy the basic demands of readers. 3 . The relationship between the translated works and the original works. In order to guarantee the translation principle of "faithfulness, elegance and expressiveness" between the translation and the original text, we must innovate and improve the translation activities through three aspects: the complete description, the artistic feelings and the outstanding styles. For example, in translating the detective novels of Conan Doyle, the translator must require the works to satisfy the thinking ability and habits of Chinese readers according to the concrete meaning of the original text and the thinking modes and habits of Chinese readers while maintaining the basic meaning of the original works, so as to improve the diverse skills of the literariness and realness of the translation literature and improve Chinese readers' reception width and depth of works.

\subsubsection{Reasonably Apply the Translation Methods}

Accurately master the translation modes of English literature works, keep improving and strengthening the translation skills. From the perspective of culturology, the translation of English literature works must be based on the reasonable mastery of the translation methods. Correctly apply the translation skills, improve the literature role and spread value of the translation works. First of all, the word-for-word translation skill. It mainly directly translates the original text and adopts the literal translation of the objects and natural landscape and directly show the original scene or object. In the translation of Notre Dame de Paris, the word-for-word translation can be adopted in the words describing Notre Dame de Paris, so that readers can better feel the holiness and majesty of Notre Dame de Paris. Second, the translation skill of conversion of part of speech. When there are some gerunds, non-finite verbs and infinitives in the English language culture that do not exist in Chinese, the conversion of part of speech has to be applied in the translation process, so as to make the works authentically translated. Finally, the additional translation and deletion. Out of the cultural differences of the Chinese and the English language, the additional translation is mainly to make proper annotation and explanation of the original text according to the meaning of the original text, so as to improve readers' comprehensive translation of the original text, such as the Naples translation case mentioned in the above text. In addition, the translation skills also involve the general translation strategy, which is mainly applied in the full-length literature works. Overall translation is conducted according to the core tenet of the original text, the core objective of cross-cultural communications, the narration structure of the story plot and the logical clue of the article progress by following the principles of the intention, acceptability and coherence. However, in the translation process, some of the key contents should be preserved, so as to guarantee the smoothness of the context.

\section{Conclusions}

Above all, the translation of literature works belongs to one of the important impetus behaviors 
in the contemporary literature development. From the perspective of the culturology, the translation of the English literature works must be influenced by the cultural background of the times and the language use habit, so as to promote the cultural obstacles of the translation work. From the perspective of culturology, the translation work of English literature works must be correlated on the basis of the translation, the translator, the original works and the author of the original works through the skills such as word-for-word literature, conversion of part of speech, additional translation or deletion. For the special literature phenomenon or the literature creation modes in the English literature works, realize the technical translation and promote the cultural spread.

\section{References}

[1] Wu Chunyu, Wang Yinquan. Research of English Translation of Cultural-loaded Words in the Huangdi's Classic from the Perspective of Eco-translatology [J]. Chinese Archives of Traditional Chinese Medicine, 2015, 24(1):61-64.

[2] Wu Yun. Construction and Recognition of Chinese Female Culture from the English Perspective-Translation Research of Female Novels in the New Time in China [J]. Chinese Translators Journal, 2015, 14(4):38-44.

[3] Meng Maoqiao. Research of Teaching Methods of English Language Literature-Review of Research of English Language Literature [J]. Journal of National Academy of Education Administration, 2015, 31(9):211-212.

[4] Chen Yamin. English and American Literature Research from the Perspective of Cultural Teaching-Review of English \& American Literature and English Teaching [J]. University Education Science, 2016, 14(5):233-234.

[5] Li Yunhua. Translation Strategy Research of Convention \& Exhibition English from the Perspective of Translation Skopos Theory-Taking the Example of Tea Fair [J]. Tea in Fujian, 2017, 39(8):252-253.

[6] Sun Ying. Exploration of Special-Purpose English Translation Practice of Beijing Opera from the Perspective of Foreign Cultural Exchange [J]. Sichuan Drama, 2016, 23(9):40-44. 